\title{
Comportamiento a la corrosión de implantes de titanio granallados*
}

\author{
C.APARICIO ${ }^{l}$, D. RODRÍGUEZ ${ }^{l}$, F.J. GIL ${ }^{l}$, \\ C. FONSECA ${ }^{2}$, M. BARBOSA ${ }^{2}$, G. NUSSBAUM ${ }^{3}$, \\ A. GARCÍA ${ }^{3}$ Y J.A. PLANELL ${ }^{1}$ \\ ${ }^{I}$ Centre de Recerca en Enginyeria Biomèdica (CREB)- \\ Dept. de Ciència dels Materials i Enginyeria Metal lúrgica. \\ Universitat Politècnica de Catalunya. Barcelona. \\ ${ }^{2}$ Instituto Nacional de Engenharia Biomédica. \\ Instituto de Biologia Molecular e Celular. \\ Porto. Portugal. \\ ${ }^{3}$ Materias Primas Abrasivas (MPA). Cornellá-Barcelona
}

\begin{abstract}
Resumen
Se ha demostrado que realizar el tratamiento de granallado sobre implantes mejora la fijación de los mismos, debido a la rugosidad superficial que adquieren. Variando el tamaño de las partículas de proyección del granallado, se varía el valor de rugosidad. Por otra parte, siempre quedan partículas adheridas en la superficie del implante, lo cual puede tener influencia en distintas propiedades, entre las cuales cabe destacar su resistencia a la corrosión. Este estudio determina el comportamiento a la corrosión, mediante mediciones potenciodinámicas, del titanio comercialmente puro granallado con diferentes materiales de partículas de proyección $\left(\mathrm{Al}_{2} \mathrm{O}_{3}\right.$ y $\left.\mathrm{SiC}\right)$ y con diferentes tamaños de partícula $(200,600$ y $900 \mu \mathrm{m}$ ). La rugosidad no tuvo influencia significativa en los resultados de corrosión, pero sí la tuvo la naturaleza de las partículas de proyección. Así, los discos tratados con SiC, debido a la oxidación de estas partículas adheridas en la superficie del titanio, presentaron densidades de corriente un orden de magnitud mayores que los discos tratados con $\mathrm{Al}_{2} \mathrm{O}_{3}$, lo que les confiere peores características de resistencia a la corrosión. No obstante, todos los discos presentaron un adecuado comportamiento frente a la corrosión.
\end{abstract}

Palabras clave: Corrosión, granallado, implante, titanio, ensayos potenciodinámicos.

\section{Introducción}

El granallado es un tratamiento mecánico superficial que en la actualidad es aplicado sobre implantes dentales de titanio comercialmente puro
(Ti c.p.) para aumentar su rugosidad superficial, ya que ello mejora su fijación en el hueso a corto y largo plazo, como se ha demostrado a través de diversos estudios in vivo ${ }^{1-3}$. Asimismo, estudios in vitro han puesto de manifiesto que la diferenciación

\footnotetext{
* Primer premio a la mejor comunicación para ponentes menores de 35 años en el XXI Symposium de la SIB (Madrid, noviembre de 1998)

Correspondencia:

Dr. F.J. Gil, Dept. Ciencia de los Materiales e Ing. Metalúrgica,

ETS Ingenieros Industriales de Barcelona, Universidad Politécnica de Catalunya,

Av. Diagonal 647, 08028 Barcelona .E-mail gil@cmem.upc.es
} 
y proliferación celular, y la producción de matriz ósea, también resultan influenciadas positivamente por el aumento de la rugosidad de la superficie $e^{4-6}$. Se ha estudiado cuál es el valor de rugosidad óptimo para optimizar la respuesta osteoblástica y la fijación ósea, variando la rugosidad a través de la variación del tamaño de las partículas de proyección en el granallado ${ }^{7-8}$.

El granallado consiste en proyectar partículas de elevada dureza a gran velocidad sobre la superficie del implante, siendo el golpeo continuo de las mismas el responsable de la deformación plástica de la superficie, la cual queda rugosa. Además, el tratamiento es adecuado porque elimina posibles defectos del mecanizado de la pieza, limpia la superficie de posibles contaminantes, y puede aumentar la vida a fatiga y resistencia a la corrosión bajo tensión del implante ${ }^{9}$.

Pero el tratamiento de granallado presenta un posible inconveniente derivado de la naturaleza química de las partículas utilizadas para ser proyectadas contra la superficie del metal a tratar, ya que debido a la alta energía en el impacto, algunas de ellas se rompen y quedan incrustadas en la superficie del implante, incluso después de su limpieza, pasivado y esterilización. Este hecho puede provocar que alguna de estas partículas puedan desprenderse y pasar a los tejidos circundantes, entorpeciendo la mineralización adecuada del hueso ${ }^{10}$ o estimulando la adhesión y diferenciación celular ${ }^{11}$, según sea la naturaleza de las partículas. Además, estas partículas pueden tener influencia en otras propiedades del implante, de entre las que cabe destacar su resistencia a la corrosión. La adhesión del material de proyección en la superficie provoca heterogeneidad físicoquímica en la misma, lo cual, evidentemente, puede ser un claro condicionante en la respuesta ante las condiciones corrosivas a las que se ve sometido un implante en el medio fisiológico.

Los estudios potenciodinámicos permiten valorar el comportamiento a la corrosión de los materiales metálicos, sometiéndolos a un potencial eléctrico variable en un fluido fisiológico a temperatura corporal. El registro de corriente eléctrica que circula por la muestra al variar el potencial al que se le somete, permite obtener información sobre la resistencia de la capa de pasivado del material, la repasivación del mismo, así como su resistencia a la corrosión localizada ("pitting") y otros aspectos derivados de su comportamiento electroquímico.

\section{Materiales y métodos}

\section{Preparación de los discos}

Se cortaron discos de Ti c.p. de Grado III de 6 $\mathrm{mm}$ de diámetro y un espesor de $2 \mathrm{~mm}$. Se prepararon discos con cada una de las siguientes superficies: sin tratamiento de granallado (ST), con tratamiento de granallado con $\mathrm{Al}_{2} \mathrm{O}_{3}$ y tamaño de partículas 200, 600 y $900 \mu \mathrm{m}$ (AL2, AL6, AL9), y con tratamiento de granallado con $\mathrm{SiC}$ y tamaño de partículas 200, 600 y $900 \mathrm{~mm}$ (SI2, SI6, SI9).

Los tratamientos de granallado, cualquiera que fuese la naturaleza o el tamaño de las partículas de proyección, se realizaron con una presión de 0,25 $\mathrm{MPa}$, durante $5 \mathrm{~s}$.

Todos los discos se lavaron, sumergidos en agua destilada y jabón neutro, durante 30 minutos en ultrasonidos. Se pasivaron con una disolución diluida de $\mathrm{HCl}$ (excepto ST) y finalmente se esterilizaron con óxido de etileno.

\section{Ensayos potenciodinámicos}

La preparación de los discos previa a los ensayos potenciodinámicos consistió en encastarlos, después de adherirlos a un hilo conductor recubierto de teflón, en una resina en frío y al vacío, para evitar alterar las propiedades del metal, así como la formación de burbujas en la resina polimérica $\left(\right.$ Epofix $^{\odot}$, Struers). La resina se dejó secar durante 24 horas.

Previo al ensayo potenciodinámico, los discos encastados se sumergieron durante dos horas en el medio y a la temperatura de ensayo, con el propósito de que el material alcanzase su potencial libre. El medio de ensayo fue Hank's balanced salt solution (HBSS, Sigma), una disolución con concentración iónica semejante a la del medio fisiológico (Tabla I), y la temperatura de ensayo fue la temperatura corporal, es decir, $37 \pm 1{ }^{\circ} \mathrm{C}$.

Los ensayos potenciodinámicos se llevaron a cabo en una celda electroquímica de vidrio de 250 $\mathrm{ml}$, con un electrodo de referencia de calomelanos, y un contraelectrodo de platino con un área de 2'3 $\mathrm{cm}^{2}$. Se utilizó un potenciostato (273 A, EG\&G) para aplicar los potenciales eléctricos. La adquisición de datos se realizó a intervalos de $1 \mathrm{~s}$.

La polarización cíclica se llevó a cabo de acuerdo con el método de ensayo propuesto por la norma ASTM G61-86 ${ }^{12}$, iniciando el ciclo a -1000 $\mathrm{mV}$ hasta $3000 \mathrm{mV}$, para acabar retornando al potencial inicial; siempre con una velocidad de 
paso de potencial de $4 \mathrm{mV} / \mathrm{s}$. Antes de iniciar el ciclo se dejó la muestra durante 5 minutos sometida al potencial de $-1000 \mathrm{mV}$, con el objetivo de evitar la desestabilización de la respuesta producida por el salto brusco de tensión al que se somete el material, ya que está previamente a su potencial libre.

Tabla I: Composición del Hank's balanced salt solution utilizado.

\begin{tabular}{lc}
\hline \multicolumn{1}{c}{ Sales Inorgánicas } & Concentración $\left(\mathbf{g}^{-\mathbf{1}}\right)$ \\
\hline $\mathrm{CaCl}_{2}$ anhidro & 0.14 \\
$\mathrm{KCl}$ & 0.40 \\
$\mathrm{KH}_{2} \mathrm{PO}_{4}$ & 0.06 \\
$\mathrm{MgCl}_{2} \cdot 6 \mathrm{H}_{2} \mathrm{O}$ & 0.10 \\
$\mathrm{MgSO}_{4} \cdot 7 \mathrm{H}_{2} \mathrm{O}$ & 0.10 \\
$\mathrm{NaCl}$ & 8.00 \\
$\mathrm{NaHCO}_{3}$ & 0.35 \\
$\mathrm{Na}_{2} \mathrm{HPO}_{4} \cdot 7 \mathrm{H}_{2} \mathrm{O}$ & 0.09 \\
Otros Componentes & \\
p-Glucosa & 1.00 \\
Rojo Fenol & 0.01 \\
\hline
\end{tabular}

\section{Resultados y discusión}

En la Tabla II se pueden consultar los resultados de rugosidad de los discos tratados con los diferentes tratamientos de granallado, obtenidos en otro estudios por los presentes autores ${ }^{7}$ Los resultados ponen de manifiesto que la variación de la naturaleza y el tamaño de las partículas de proyección alteran el valor de la rugosidad superficial significativamente.

Tabla II: Valores medios de rugosidad superficial $\left(\mathrm{R}_{\mathrm{a}}\right.$ : media aritmética de las desviaciones del perfil) de los discos de Ti c.p. granallados con distintos tipos de material y tamaño de partículas de proyección ${ }^{7}$. D.E.: desviación estándard.

\begin{tabular}{lc}
\hline & R $_{\mathbf{a}} \pm$ D.E. $(\mu \mathbf{m})$ \\
\hline ST & $0.33 \pm 0.11$ \\
AL2 & $1.82 \pm 0.03$ \\
AL6 & $4.34 \pm 0.16$ \\
AL9 & $6.84 \pm 0.60$ \\
SI2 & $2.24 \pm 0.15$ \\
SI6 & $5.00 \pm 0.67$ \\
SI9 & $7.39 \pm 0.26$ \\
\hline
\end{tabular}

En las Figuras 1 y 2, se pueden observar micrografías de la superficie de un disco AL6 y de un disco SI6, respectivamente; obtenidas en un estudio previo de los presentes autores ${ }^{11}$. Se puede comprobar que, tanto en el caso del óxido de aluminio como en el del carburo de silicio, quedan partículas adheridas sobre la superficie del titanio, incluso después de su lavado, pasivado y esterilizado.

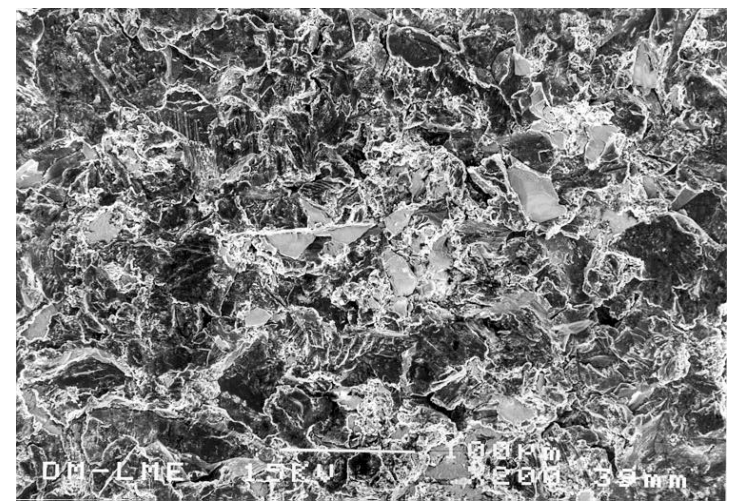

Figura 1: Micrografía de la superficie de un disco de titanio granallado con partículas de $\mathrm{Al}_{2} \mathrm{O}_{3}$ de tamaño $600 \mu \mathrm{m}$ (AL6) ${ }^{11}$

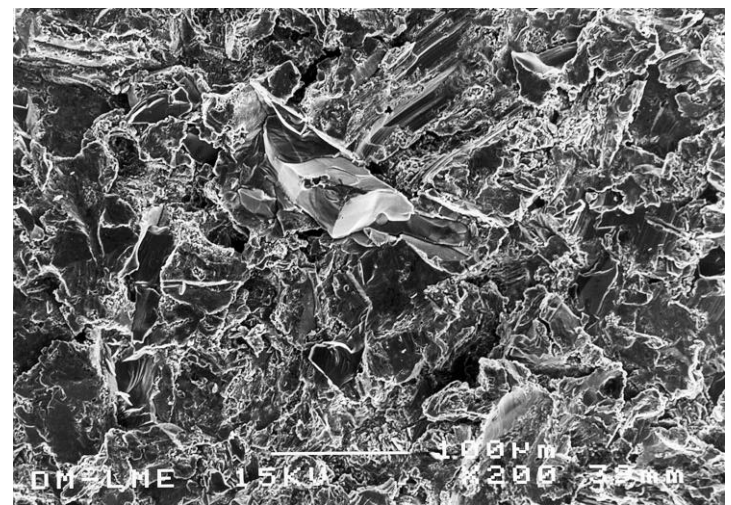

Figura 2: Micrografía de la superficie de un disco de titanio granallado con partículas de $\mathrm{SiC}$ de tamaño $600 \mu \mathrm{m}$ (SI6) ${ }^{11}$.

En la Figura 3 está representado el ciclo de polarización obtenido al ensayar el disco ST. Se puede comprobar que el comportamiento es de un material muy estable, formando una capa de pasivación dinámica a partir de $-200 \mathrm{mV}$, estabilizándose la densidad de corriente que circula a través del Ti c.p. alrededor de $15-20 \mu \mathrm{A} / \mathrm{cm}^{2}$.

En las Figuras 4 y 5 se pueden consultar los ciclos de polarización para los discos de Ti c.p. granallados con los tres diferentes tamaños de partícula de $\mathrm{Al}_{2} \mathrm{O}_{3}$ (AL2, AL6, AL9) y $\mathrm{SiC}$ (SI2, SI6, SI9), respectivamente.

El comportamiento general no se ve modificado al variar el tamaño de partícula de proyección, tanto si éstas son de $\mathrm{Al}_{2} \mathrm{O}_{3}$ como si son de $\mathrm{SiC}$. Únicamente se aprecia una tendencia a aumentar la densidad de corriente al aumentar el tamaño de las partículas. Esta tendencia sólo se ve alterada al comparar los resultados entre AL6 y AL9, ya que los valores de densidad de corriente no son 
significativamente superiores para la muestra más rugosa. Sin embargo, hay que tener en cuenta que la curva del disco AL9 presenta múltiples pequeñas inestabilidades. Este hecho, que indica que se produjo ruido eléctrico durante el ensayo potenciodinámico, provoca interferencias en la determinación de los valores de densidad de corriente. En consecuencia, este resultado anómalo puede estar directamente relacionado con el ruido eléctrico registrado.

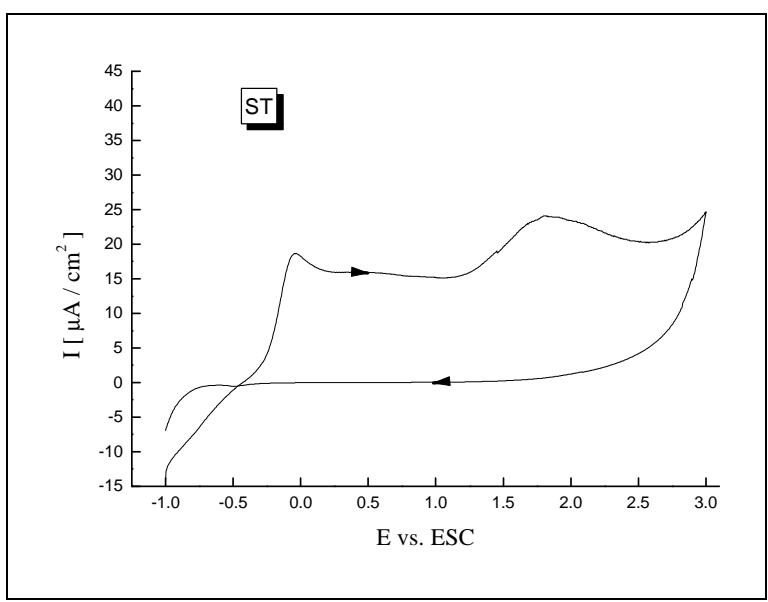

Figura 3: Curva de polarización cíclica para un disco de Ti c.p. sin tratamiento de granallado (ST).

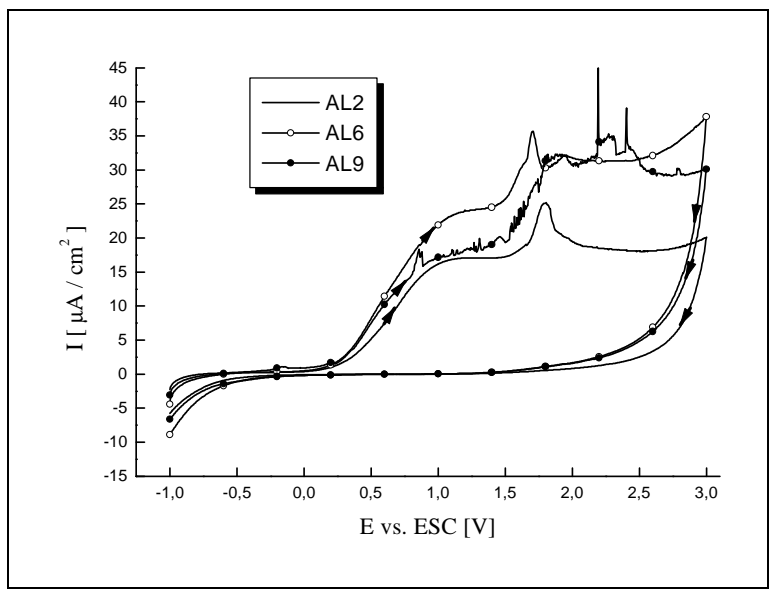

Figura 4: Curvas de polarización cíclica para discos de Ti c.p. granallados con partícula de $\mathrm{Al}_{2} \mathrm{O}_{3}$ de tamaño $200 \mu \mathrm{m}$ (AL2), $600 \mu \mathrm{m}$ (AL6) y $900 \mu \mathrm{m}$ (AL9).

El resultado obtenido consistente en que al aumentar el tamaño de las partículas de proyección aumenta la densidad de corriente eléctrica que circula por el material no implica un empeoramiento del comportamiento a la corrosión del mismo. Esto es así porque los resultados de densidad obtenidos deberían estar corregidos ya que están calculados con el valor del área aparente de los discos. El aumento del tamaño de partícula supone aumento de rugosidad (Tabla I), lo cual implica una mayor área efectiva de paso de corriente por la muestra, y por lo tanto, si los valores de densidad de corriente estuvieron corregidos, calculándolos con el valor de su área efectiva en lugar de con su área aparente, serían inferiores a los representados en las curvas. Así pues, se puede decir que el tamaño de partícula no influye en la densidad de corrosión, aunque sí ligeramente en la resistencia a la corrosión del implante en servicio debido al aumento de superficie que supone el aumento de rugosidad. Si la superficie aumenta y la densidad de corriente permanece constante la cantidad total de corriente eléctrica circulando a través del titanio irá ligeramente aumentando al aumentar el tamaño de las partículas de proyección.

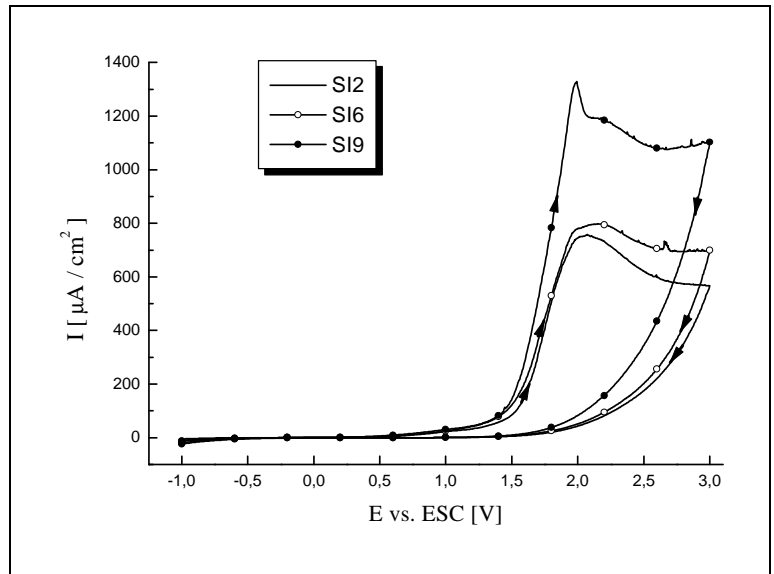

Figura 5: Curvas de polarización cíclica para discos de Ti c.p. granallados con partícula de SiC de tamaño $200 \mu \mathrm{m}$ (SI2), $600 \mu \mathrm{m}$ (SI6) y $900 \mu \mathrm{m}$ (SI9).

El material de las partículas de proyección sí tiene distinta influencia sobre la respuesta potenciodinámica del Ti c.p. granallado. Por su parte, los discos granallados con $\mathrm{Al}_{2} \mathrm{O}_{3}$ presentan un comportamiento muy similar al ST. La única diferencia significativa reside en que en los discos AL2, AL6 y AL9 la capa protectora del metal rompe (momento en que aumentan la densidad de corrriente) a valores de tensión superiores (aprox. $300 \mathrm{mV}$ ) que en el caso del titanio sin granallar (aprox. -100 mV), lo cual implica un carácter más noble por parte de los discos granallados con $\mathrm{Al}_{2} \mathrm{O}_{3}$. Sin embargo, este resultado se puede explicar por el hecho de que los discos granallados se pasivaron 
con una disolución de $\mathrm{HCl}$, mientras que el titanio sin granallar no se pasivó. La pasivación aumenta el grosor del óxido de titanio que recubre al metal, lo cual lo ennoblece. Por lo tanto, no se puede decir que las partículas de $\mathrm{Al}_{2} \mathrm{O}_{3}$ influyan en la respuesta potenciodinámica. De hecho, los valores de densidad de corriente que alcanza la muestra AL2, la menos rugosa de las granalladas con $\mathrm{Al}_{2} \mathrm{O}_{3}$, al estabilizarse la capa dinámica son del mismo valor que para la muestra sin granallar $\left(15-20 \mu \mathrm{A} / \mathrm{cm}^{2}\right)$, e incluso, el pico de densidad de corriente que se observa para estos discos granallados, alrededor de $1600 \mathrm{mV}$, también aparece en el disco ST, lo cual indica que probablemente se debe a la oxidación a estados superiores del óxido superficial del titanio y no a cualquier otro aspecto relacionado con la presencia en la superficie de las partículas de $\mathrm{Al}_{2} \mathrm{O}_{3}$.

Por otra parte, si se comparan directamente las curvas obtenidas para los discos granallados con $\mathrm{SiC}$ o $\mathrm{Al}_{2} \mathrm{O}_{3}$ con un mismo tamaño de partícula, un ejemplo del cual lo tenemos en la Figura 6 donde se comparan AL2 y SI2, se aprecia que la respuesta a la polarización presenta diferencias notables, ya que los valores de densidad de corriente que circulan a través de los discos granallados con $\mathrm{SiC}$ (650-1300 $\mu \mathrm{A} / \mathrm{cm}^{2}$ ) son algo más de un orden de magnitud superiores a las que circulan por los discos granallados con $\mathrm{Al}_{2} \mathrm{O}_{3}\left(15-30 \mu \mathrm{A} / \mathrm{cm}^{2}\right)$.

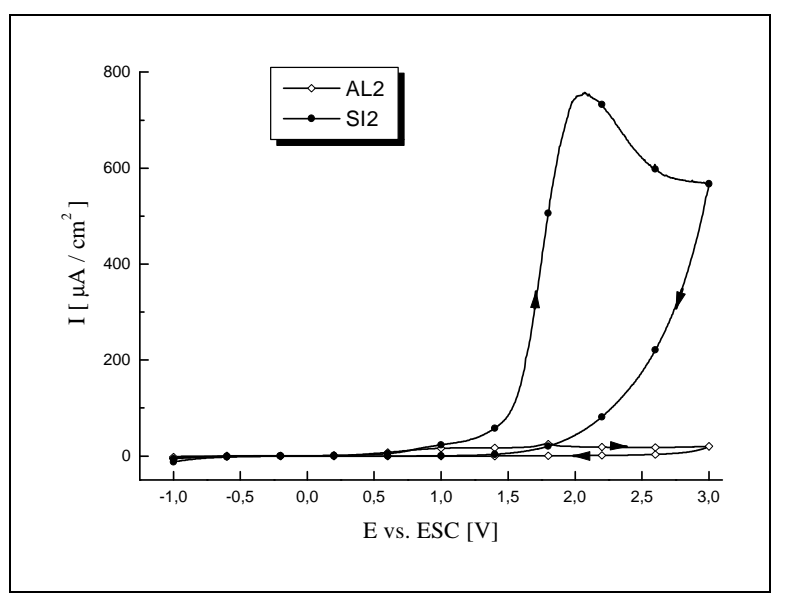

Figura 6 : Curvas de polarización cíclica para discos de Ti c.p. granallados con partículas de proyección de $200 \mu \mathrm{m}$ de $\mathrm{Al}_{2} \mathrm{O}_{3}$ (AL2) y de $\mathrm{SiC}$ (SI2).

Este hecho está probablemente relacionado con la oxidación de las partículas de $\mathrm{SiC}$, la cual comienza sobre los $1000 \mathrm{mV}$, momento en que las dos curvas de polarización de la figura 6 se separan. Cuando terminan la oxidación (aprox. $1900 \mathrm{mV}$ ) los valores de densidad de corriente caen a valores inferiores paulatinamente. Todo ello, implica que los discos granallados con $\mathrm{SiC}$ presentan una resistencia a la corrosión inferior a los granallados con $\mathrm{Al}_{2} \mathrm{O}_{3}$, debido a la menor estabilidad electroquímica de las partículas de $\mathrm{SiC}$ que quedan adheridas sobre la superficie del Ti c.p. No obstante, los valores de corriente que circulan por los discos granallados con $\mathrm{SiC}$ son también de valor pequeño, lo cual indica que su resistencia a la corrosión es adecuada.

Por último, cabe comentar la alta capacidad de repasivación de todos los discos ensayados ya que las curvas presentan una gran pendiente al iniciar el retorno en el ciclo de polarización desde los 3000 $\mathrm{mV}$. Este resultado pone de manifiesto un nuevo comportamiento satisfactorio del titanio granallado con $\mathrm{Al}_{2} \mathrm{O}_{3}$ y $\mathrm{SiC}$, atendiendo a las solicitaciones electroquímicas a las que se puede ver sometido en servicio.

\section{Conclusiones}

A pesar de las diferencias encontradas en el comportamiento electroquímico del titanio granallado, en función de la naturaleza química de las partículas de proyección utilizadas en el tratamiento superficial, todos los discos ensayados presentaron un adecuado comportamiento frente a la corrosión, lo cual implica que el granallado con $\mathrm{Al}_{2} \mathrm{O}_{3}$ y SiC sobre Ti c.p. es un tratamiento que no perjudica de forma significativa la respuesta electroquímica de los implantes.

\section{Bibliografía}

1. Predecki P., Stephan J.E., Auslaender B.A., Mooney V.L., Kirkland K. Kinetics of bone growth into cylindrical channels in aluminium oxide and titanium. J Biomed Mater Res 1972; 6: 375-400.

2. Buser D., Schenk R.K., Steineman S., Fiorelini J.P., Fox C.H., Stich H. Influence of surface characteristics on bone integration of titanium implants. A histomorphometric study in miniature pigs. J Biomed Mater Res 1991; 25: 889-902.

3. Johansson C.B., Wenneberg A., Han C.-H., Albrektsson T. A quantitative comparison of titanium implants, en Fifth World Biomaterials Congress, Toronto June 1996, p. 478.

4. Bowers K.T., Keller J.C., Michaels C.M. Optimitazion of surface micromorphology for enhanced osteoblast response in vitro. Int J Oral Maxillofac Implants 1992; 7: 302.

5. Groessner-Schreiber B., Tuan R.S. Enhanced extracellular matrix production and mineralitazion by osteoblasts cultured on titanium surfaces in vitro. 
J Cell Sci 1992; 101: 209.

6. Martin J.Y., Schwartz Z., Hummert T.W., Schraub D.M., Simpson J., Lankford Jr J, y col. Effect of titanium surface roughness on proliferation, differentiation, and protein synthesis of human osteoblast-like cells. J Biomed Mater Res 1995; 29: 389-401.

7. Aparicio C, Gil F.J., Peraire C., Padrós A., Planell J.A. Osteoblast cell response to commercially pure titanium implants blasted with different particles, en The $9^{\text {th }}$ World Conference on Titanium, Saint Petersburg June 1999, Aceptado.

8. Wenneberg A., Albrektsson T., Andersson B. An animal study of c.p. titanium screws with different surface topographies. J Mater Sci Mater Med 1995; 6: 302-309.

9. Oshida Y., Sachdeva R., Miyazaki S., Daly J. Effects of shot peening on surface contact angle of biomaterials. 1993 J Mater Sci Mater Med; 4: 443447.

10. Gross U., Strunz V. The interface of various glasses and glass ceramics with bony implantation bed. 1985; J Biomed Mater Res; 19: 251-271.

11. Aparicio C., Gil F.J., Padrós A., Peraire C., Planell J.A. Influencia de la naturaleza de las partículas de proyección en el proceso de shot peening en la mejora de la osteointegración de implantes dentales. 1997; Biomecánica; 9: 92-100.

12. ASTM G61-86, Standard Test Method for Conducting Cyclic Potentiodynamic Polaritazion Measurements for Localized Corrosion Susceptibility of Iron, Nickel, or Cobalt Based Alloys. 1989; Annual Book of ASTM Standards, Vol. 0302. Philadelphia (PA), American Society for Testing and Materials; 345-361. 